\title{
Text Document Clustering with Negative Noun Attributes
}

\author{
S. Vijayalakshmi ${ }^{1}$ and P. Murugeswari ${ }^{2}$ \\ ${ }^{1}$ Department of Computer Applications, NMS S. Vellaichamy Nadar \\ College, Madurai,Tamilnadu, India. \\ ${ }^{2}$ Department of Computer Science and Engineering, Karpagam College \\ of Engineering, Coimbatore, Tamil Nadu, India. \\ Corresponding author email: pandyviji@gmail.com
}

\begin{abstract}
High-dimensional feature space is the biggest challenge for all its mining duties. People who used automatic document representation methods based on statistics and information theory had dealt with the issue of high dimensions. The efficiency of HCL K-Means clustering and clustering with Flocking algorithm was analysed in this article with noun characteristics chosen by chi-square and CHIR techniques with positive and negative noun characteristics. It was noted that the clustering efficiency rises with the noun attributes with favourable noun characteristics selected by the CHIR techniques.
\end{abstract}

\section{KEY WORDS: NOUN ATTRIBUTES, CHI, CHIR, HCLK-MEAN AND FLOCKING ALGORITHM.}

\section{INTRODUCTION}

Representation of bags of nouns is a vector of unordered set of noun word information in the document. The vectors can be either numerical or Boolean. A Boolean vector indicates the existence or lack of each noun phrase in a document. Numerical value in the vector indicates the strength of the calculated noun word based on its frequency, theory of information or statistics. The representation has been commonly used in the processing of natural language and in the retrieval of data. The drawback of BOW illustration is that the context of words, occurrences, and order of occurrences of words is neglected.The drawback of BOW representation is that it ignores the context of the words, place of occurrences and order of occurrences of the words (Asha et al., 2010).

Still, this is the most widely used document representation, but $\mathrm{BoN}$ is used to extract the noun word and it brings the concept and shows good clustering results. Yang and Pedersen , made a comparative study on the feature selection techniques proposed for text classification and

Biosc Biotech Res Comm P-ISSN: 0974-6455 E-ISSN: 2321-4007

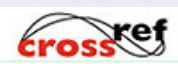

Identifiers and Pagination

Year: 2021 Vol: 14 No (9) Special Issue

Pages: 277-284

This is an open access article under Creative

Commons License Attribn 4.0 Intl (CC-BY). DOI: http://dx.doi.org/10.21786/bbrc/14.9.52 reported that the features chosen based on the $x^{\wedge}$ value are better than other techniques. CHIR is a variant of chisquare method proposed by Li et al, which consider the relevancy of feature to the category for feature selection (Abusamra 2013).

Related Work: The term discrimination model of Salton provides significance to the depiction of text by specifying a good text attribute as the one that improves the average range of all pairs of document vectors. Bag of words is the most common representation of documents Significant improvements were achieved by Tzeras and Hartmann[4] using noun phrases acquired by combining syntactic and statistical criteria, where a statistical filter complemented the "crude" syntactic technique.Only syntactic sentences in the category were maintained that happened at least three times. Its high-dimensional word space is the most difficult problem in text clustering (Huiqing et al., 2002).

Mitra et al used new indexes to evaluate similarity of attributes in order to detect redundancy attributes. Huiqing Liu et al conducts a comparative research using distinct methods of choice of features such as entropy, Chi Squared method, T-Statistics and MIT correlation and correlation-based technique of choice of features using Profiles of Gene Expression and Proteomic Patterns. He suggested extracted characteristics significantly enhance the classifier's efficiency. Tao Liu et al suggested a new technique of selecting attributes, called Term Contribution,
Article Information

Received: $28^{\text {th }}$ Apr 2021 ccepted after revision: $15^{\text {th }}$ June 2021 
which ranks the attribute through its full contribution to the similarity of records in a data set.

Figure 1.1 Examples for BON Representation

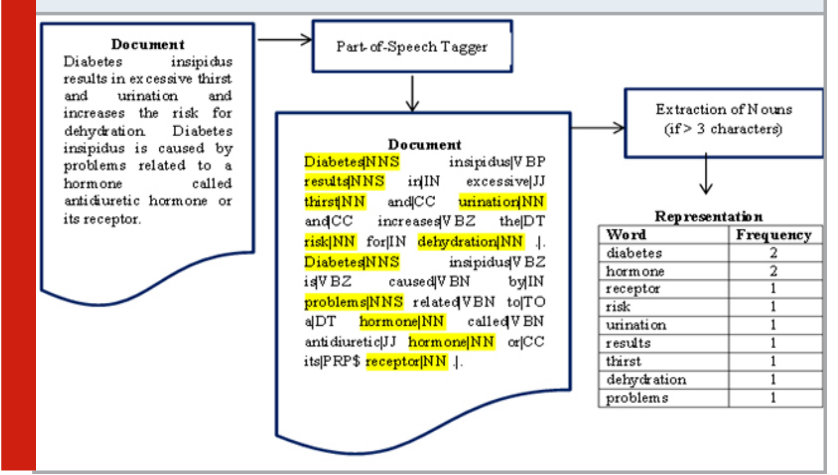

Yi Hong et al suggested an unsupervised feature selection algorithm called Guided unsupervised Ensembles feature selection algorithm and is dimension-unbiased. Yanjun Li et al launched a feature selection technique called CHIR to evaluate the word goodness. Ridvan Saracoglu et al explore the document belonging to more than one category and used to determine the records belonging to which categories. Fuzzy similarity $(\alpha-F C M)$ and multiple categories vector method (MCVM) to their objectives. Asha Gowda Karegowda et al uses two filter approaches, namely Gain ratio and Correlation-based feature selection methods used to elucidate the significance of feature subset selection for Pima Indian Diabetic Database (PIDD) classification, and its experimental results show that the information gain method provides better classification accuracy. It is also shown that by using a feature selection method, classification precision can be considerably improved by a tiny amount of genes.

\subsection{Bag of Nouns (Bon) Model:}

Documents are represented as dimensionality vectors in this representation, which is equal to the number of noun words in the training corpus. In the training corpus, each component in the vector reflects a noun phrase. Figure 1.1 demonstrates an illustration of the building of the Noun Index. It can be noted that the document contains 30 phrases. In the representation model of Bag-of-Nouns (BoN), 11 words (37.67\%) can be obtained as Nouns using RiTa WordNet ontology and can be included in the list of characteristics as shown in the table (Figure 1.1). Only nouns are considered in this article to decrease their dimension. Clustering is not able to perform well in a high dimension. Clustering is not able to perform well in a high dimension. All the word in the document is not important and some are irrelevant and redundant.

1.3 Attribute Selection Methods Used: This article discusses some of the techniques of choosing existing attributes used in this study (Mitra \&t Murthy 2002).

\subsubsection{The Concept of $\chi^{2}$ Statistic (CHI)}

The $\chi^{2}$ term-category independence test is a popular attribute selection technique for text mining retrieval problems. This is a two sided attribute selection metric. $\chi^{2}$ value for a term is obtained by comparing the observed frequencies with expected frequencies, when the occurrence of the terms is assumed to be independent. In a corpus that contains $n$ labelled documents, which fall in $m$ categories, Noun (N) X Document (D) contingency table is formed for each term (Jinxin et al., 2010).

Expected frequency E (i,j), where i represents the presence or absence of an attribute and $\mathrm{j}$ represents whether the document belongs to a category, calculated using Equation (1.1).

$$
E(i, j)=\frac{\sum_{a \in(N 1, N 2)} O(a, j) \sum_{b \in(D 1, D 2)} O(i, b)}{n}
$$

$\chi^{2}$ Value for a noun words (N) in the training corpus, with respect to the document (D) is defined in the Equation (1.2):

$$
\chi_{n, d}^{2}=\sum_{i \in(N 1, N 2)} \sum_{j \in(D 1, D 2)} \frac{(O(i, j)-E(i, j))^{2}}{E(i, j)}
$$

In statistics, "degree of freedom" is used to describe the number of values in the final calculation of a statistic that are free to vary. The "degree of freedom" for a contingency table of dimension is ( $\mathrm{r}-1) \mathrm{X}(\mathrm{c}-1)$. The value obtained with Equation (1.2) is compared with the value in the standard $\chi^{2}$ tabulation for the determined degrees of freedom with a confidence level.

Null hypothesis has to be rejected when the $\chi_{n, d}^{2}$ calculated using Equation (1.2) is greater than the tabulation and alternative hypothesis has to consider. When it is assumed that there is a dependency goodof-fit is used to decide the dependency. Measures of the goodness of fit typically summarize between the observed values and expected values under the model in question. For a corpus with $m$ classes, term goodness is calculated as either the average as defined in Equation (1.3) or the maximum as defined in Equation 1.4

$$
\begin{gathered}
\chi_{\text {avg }}^{2}(W)=\sum_{j=1}^{m} p\left(c_{j}\right) \chi_{n, d_{j}}^{2} \\
\chi_{\max }^{2}(W)=\sum_{j=1}^{m} \max _{j}\left\{\chi_{n, d_{j}}^{2}\right\}
\end{gathered}
$$

$p\left(c_{j}\right)$ is the probability of documents to be in the category $c_{\dot{f}}$. In this research, maximum value is used for comparison. Noun terms were ranked by their $\chi^{2}$ value and terms having strong dependency to the categories were selected as attributes (Meedeniya et al., 2009).

1.3.1.1. Drawback of $\chi^{2}$ Statistics: CHI algorithm determines only whether there is dependency between term and a category and not the type of dependency. Hence $\chi^{2}$ value does not distinguish positive and negative attributes of a document. Li et al varied by the $\mathrm{CHI}$ method by introducing the relevancy of the term to a 
document and disguised the attributes into positive and negative (Ridvan 2008).

1.3.2. The Concept Of CHIR Method: $\mathrm{CHIR}$ is a supervised attribute selection algorithm based on $\chi^{2}$ statistics, which not only determines whether there is a dependency between noun and a document but also the type of dependency. To determine the type of dependency, a new relevancy measure $\mathrm{R}_{\mathrm{n}, \mathrm{d}}$ is defined in CHIR as shown in Equation (1.5):

$$
R_{n, d}=\frac{O(n, d)}{E(n, d)}
$$

If there is no dependency between the noun $\mathrm{n}$ and the documents $d$, then the value of $R_{n, d}$ is close to 1 . If there is a positive dependency then the observed the frequency is larger than the expected frequency, hence value of $R_{n, d}$ is larger than 1 and when there is a negative dependency $\mathrm{R}_{\mathrm{n}, \mathrm{d}}$ is smaller than 1 . Based on $\chi^{2}$ statistics and $\mathrm{R}_{\mathrm{n}, \mathrm{d}}$ a new definition for term goodness for corpus with $\mathrm{m}$ classes in given in Equation (1.6):

$$
r \chi_{n, d}^{2}(n)=\sum_{j=1}^{m} p\left(R_{n, d_{j}}\right) \chi_{n, d_{j}}^{2} \text { wit } \square R_{n, d_{j}}>1
$$

Wherep $\left(R_{n, d j}\right)$ is the weight of $\chi_{n, d, j}^{2}$ in the corpus. In terms of $R_{n, d, j}, \quad p R_{n, d, j}$ is defined as Equation (1.7):

$$
p\left(R_{n, d_{j}}\right)=\frac{R_{n, d_{j}}}{\sum_{j=1}^{m} R_{n, d_{j}}} \text { wit } \square R_{n, d_{j}}>1
$$

Larger value of $r \chi^{\wedge 2}(n)$ indicates that the noun word is more relevant to the document. The attribute selection procedure first calculates $r x^{\wedge}$ value for each unique noun word in the corpus, sorts them in descending order based on their $r \chi^{2}$ value and then selects the top ' $\mathrm{q}$ ' terms. In this research, same number of attributes was selected for each document (Vijayalakshmi \&t Manimegalai 2014).

1.4 Algorithm to Select Positive Words Using Chir: The dataset includes records such as news article, multiple high court judgments, and discussion group posts. They comprise a lot of metadata like date, location, writer, and so on. Therefore, by removing such meta data, the first stage in the algorithm is to clean up the benchmarks. All papers contain non-clustering phrases such as articles and prepositions. Hence, Stanford University's listed stop words (571) were removed from the training corpus. Stemming is the method by which derived words are reduced to their root form. The phrases like "stemmer","stemming", and stemmed, for instance, are based on the root term "stemmer". Since 1968, several researches on the stemming of phrases in English can be carried out (Vijayalakshmi \&t Manimegalai 2013.

No stemming algorithm does a ideal stemming; either by streaming or by streaming a phrase. The most common porter stemming algorithm is used in these studies. In the stemming algorithm, there are some minor mistakes such as; for certain words, it provides more than one stem. For example, the algorithm "communicate" for the word "communicates" gives two stems. By selecting only the terms that appear in the dictionary, these mistakes can be eliminated.

Contingency table is built for each unique word in the training corpus and $\mathrm{R}_{\mathrm{n}, \mathrm{d}}$, the relevancy of the noun word to document $d$ is determined. Words with $R(n, d)$ value less than 1 are eliminated and noun - goodness $\left(\mathrm{r} \chi^{2}\right)$ is calculated for all positive words using Equation (1.6). Then the noun words are sorted based on their $\chi^{2} 2$ value and the top 'p' words are chosen as features.

1.5 Time Complexity of Chir for Bon Representation: The suggested algorithm pre-processes the records and selects from the training corpus unique non-stop nouns. The algorithm that uses BoN representation to depict records, the first step is to link to WordNet to determine the vocabulary POS in the training corpus. This algorithm's complexity is $\mathrm{O}(\mathrm{V})$, where $\mathrm{V}$ is the amount of vocabulary terms. The CHIR algorithm is used to calculate its relevance score for each noun. The complexity of the entire selection algorithm for the attribute is therefore $\left(\mathrm{V}+\mathrm{V}^{*} \mathrm{~m}+\mathrm{V}^{*} \log \mathrm{V}\right)$, where $\mathrm{m}$ is the amount of documents in the database. The word $\mathrm{V}$ may be ignored because, compared to $\mathrm{V}^{*} \mathrm{~m}$, it has less development.The simplified complexity of the algorithm is $\mathrm{O}\left(\mathrm{V}^{*} \mathrm{~m}+\mathrm{V}^{*} \log \mathrm{V}\right)$.

1.6 Experimental Setup: The tests were performed with a single Intel core i5 duo processor with 2.40 GHz velocity, 4 GB RAM, and 64-bit operating system from Windows7. WordNet was linked to the English dictionary during testing to understand the phrases. Experiments with randomly selected training papers were performed 10 times. The K-Means algorithm based on Hard CompetitiveLearning and flocking algorithm clustering were used for testing. The findings shown in this paper's tables and numbers are the average clustering performance during testing.The clustering performance was first analysed by selecting the top ' $q$ ' positive characteristics and then included in addition to the positive characteristics few appropriate adverse characteristics of the document

1.6.1 Connecting to Wordnet: RiTa WordNet is intended to be a user-friendly toolkit for natural language and generative literature experiments. RiTa is introduced with a single API in Java and JavaScript and integrated with Processing as an option. This study utilizes Javabased RiTa WordNet. It is free / free and open-source through a GPL license. JWNL (Java WordNet Library) is a WordNet dictionary access Java API. It groups English words into Noun, verb, adjective, adverb, etc., gives brief, general definition, and documents the different semantic relationships between these sentences. This grouping helps to readily use WordNet for automatic text assessment and implementation of artificial intelligence (Salton et al., 1975; Tao et al., 2003). 


\subsubsection{Data Sets Used for Experimentation:}

Table 1.1 Summary of data sets used in experiments

\begin{tabular}{|l|c|c|c|}
\hline Dataset & $\begin{array}{c}\text { No. Of. Docs } \\
\text { in Dataset }\end{array}$ & Classes & $\begin{array}{c}\text { Dataset } \\
\text { size }\end{array}$ \\
\hline DS1 & 5000 & 5 & $11.3 \mathrm{MB}$ \\
\hline DS2 & 5000 & 5 & $9.91 \mathrm{MB}$ \\
\hline Reuter & 5000 & 5 & $8.61 \mathrm{MB}$ \\
\hline SCJC & 500 & 5 & $4.07 \mathrm{MB}$ \\
\hline
\end{tabular}

This research work utilizes four distinct datasets created from the two common text clustering benchmarks 20Newsgroups, Reuter-21578, and Specific Crime Judgement Corpus (SCJC). The First dataset (DS1) is made up of five types of documents: alt.atheism, sci. med, sci.space, rec.sport.hockey, comp.windows.x of 20Newsgroup. The second dataset (DS2) is made up of five classifications of documents: alt.atheism, comp. graphics, comp.sys.ibm.pc.hardware, comp.sys.mac. hardware, comp.windows.x. It can be noted that the subjects of the first dataset are distinct while computer science is associated with four subjects out of five in DS2. Documents in both alt.atheism classifications are commonly used in both datasets. The third dataset (Reuter) consisted of five classifications of documents: acquisition, crude, earnings, grain, money-fx of Reuter 21578 (Tzeras \&t Hartmann 1993).

Finally, the fourth dataset is the Specific Crime Judgment Corpus (SCJC) synthetic dataset. It involves five categories: offence against state, offence against property, offence against human body, offence relating to religion, offence relating to marriage. There are 100 true judgments in each category. These judgments are gathered at distinct phases of time from the internet and have been classified by human specialists and grouped manually into five classifications. Table 1.1 lists the entire corpus used for this research. In this research, 50\% of the corpus is considered to be a training dataset and is used for extraction of noun attributes and the remaining $50 \%$ is used to test data sets. The test dataset is used to assess the quality of BON representation characteristics and the quality of the cluster (Yanjun et al., 2008).

\section{MATERIAL AND METHODS}

The characteristics chosen by the suggested noun document representation algorithm were assessed using well-known clustering metrics such as precision, recall, F- Measure, entropy, purity, overall F-Measure, overall Entropy, and overall purity (Yanjun et al., 2008).

\section{RESULTS AND DISCUSSION}

This section describes the outcomes of tests performed using noun-document representation with and without negative attributes for different attribute selection algorithms.
1.8.1 Results of The CHI, CHIR, and CHIR NEG With HCLK-Mean Clustering Algorithm: The HCLK-Mean clustering's high precision shows that all returned by clustering was a appropriate outcome. But high precision at low rates of recall shows that not all appropriate items could have been discovered by the cluster. Table 1.2 and Table 1.3 illustrate the precision and recall of the clustering of HCLK-Means for separate datasets of distinct sizes when experimenting with attributes selected for BON representation by various attribute selection methods.Clustering precision and recall improved with the positive attributes chosen by the CHIR technique. When negative features were included in the space attribute, a decrease in the metrics was noted. It was noted that, when negative attributes were included, there was a slight rise in accuracy than CHI.

Table 1.2 Comparison of precision of HCLK-Mean clustering with CHI, CHIR, and CHIR NEG methods for text various datasets

\begin{tabular}{|l|c|c|c|}
\hline Dataset & CHI & CHIR & CHIR NEG \\
\hline DS1 & 0.73 & 0.99 & 0.79 \\
\hline DS2 & 0.71 & 0.84 & 0.81 \\
\hline Reuter & 0.66 & 0.88 & 0.87 \\
\hline SCJC & 0.74 & 0.61 & 0.60 \\
\hline
\end{tabular}

Table 1.3 Comparison of recall of HCLK-Mean clustering with CHI, CHIR, and CHIR NEG methods for text various datasets

\begin{tabular}{|l|c|c|c|}
\hline Dataset & CHI & CHIR & CHIR NEG \\
\hline DS1 & 0.83 & 0.99 & 0.41 \\
\hline DS2 & 0.75 & 0.93 & 0.68 \\
\hline Reuter & 0.98 & 0.89 & 0.88 \\
\hline SCJC & 0.67 & 0.79 & 0.68 \\
\hline
\end{tabular}

Figure 1.2 demonstrates the HCLK-Mean clustering F-measure with different attribute selection techniques for the different datasets. When selected attributes were observed, it was observed that nouns are related to the dataset to the attributes selected for the different datasets with CHIR. That's why clustering efficiency was always high for all datasets. Attributes selected from datasets were more general terms and were unable to efficiently discriminate against documents (Hong et al., 2007).

Figure 1.3 displays overall HCLK-Mean clustering F-measure with CHI, CHIR, and CHIR NEG techniques for different datasets of text. The clustering algorithm HCLK-Mean could efficiently cluster the files as each dataset must have distinctive noun phrase for each dataset category. An increase in Overall F-Measure with all data set with CHIR method's positive attributes. 
Figure 1.2 Comparison of F-Measure of HCLK-Mean clustering with CHI, CHIR, and CHIR NEG methods for text various datasets

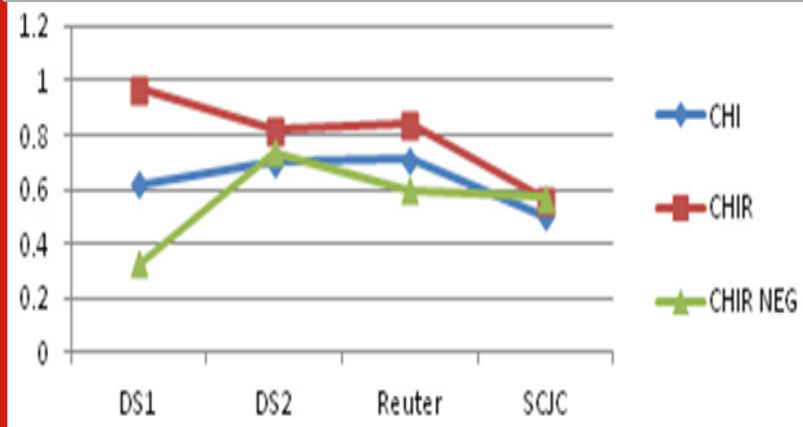

Figure 1.3 Comparison of overall F-Measure of HCLKMean clustering with CHI, CHIR, and CHIR NEG methods for text various datasets

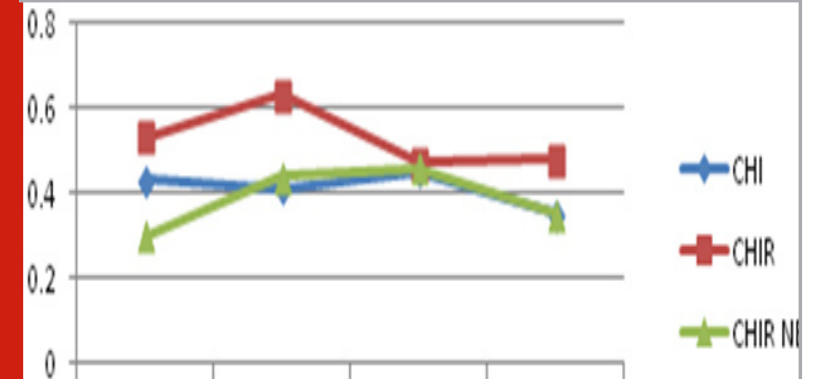

DS1 DS2 Reuter SOS

Figure 1.4 Comparison of purity of HCLK-Mean clustering with CHI, CHIR, and CHIR NEG methods for text various datasets

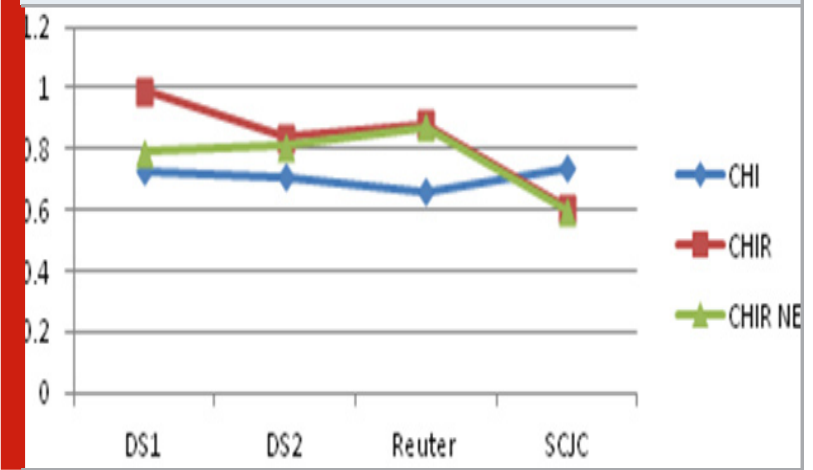

Figure 1.4 shows the comparative results of Purity of the HCLK-Means clustering for various datasets. The HCLK-Means clustering was able to effectively cluster the documents of the categories alt.atheism, comp. graphics, comp.sys.ibm.pc.hardware, comp.sys.mac. hardware, comp.windows.x in DS2 dataset. Analysis of attribute chosen by the CHIR method showed that there were number of common noun words between the above categories (Yiming et al., 1997).
Figure 1.5 Comparison of overall entropy of HCLK-Mean clustering with CHI, CHIR, and CHIR NEG methods for text various datasets

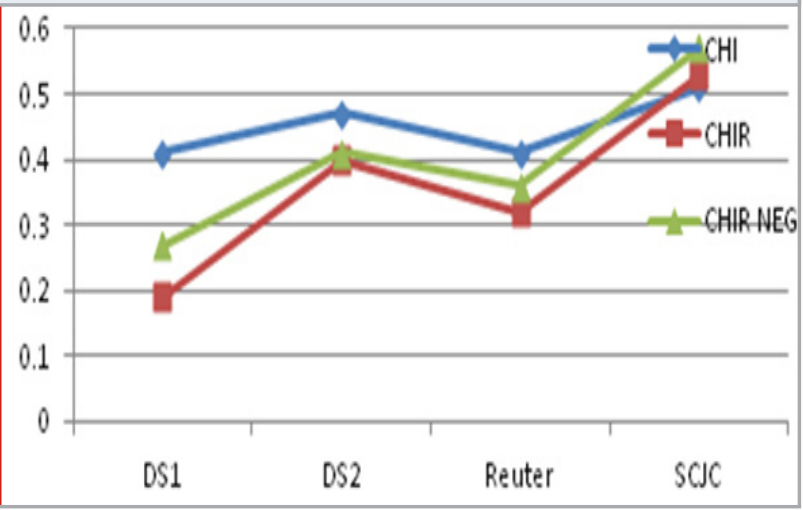

Figure 1.6 Comparison of F-Measure of Flocking-based clustering with CHI, CHIR, and CHIR NEG methods for text various datasets

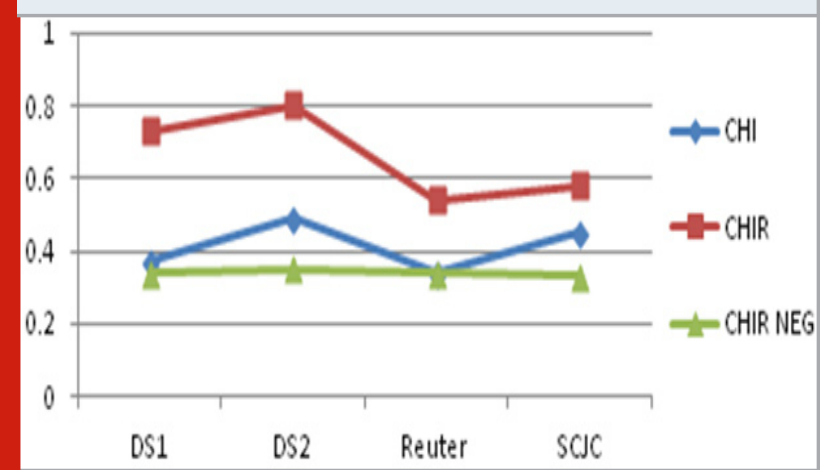

Figure 1.7 Comparison of overall F-Measure of Flockingbased clustering with CHI, CHIR, and CHIR NEG methods for text various datasets

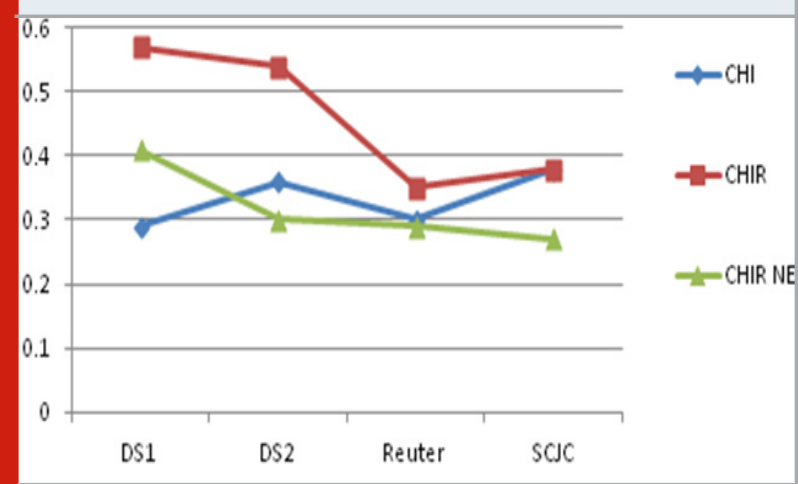

The overall purity of the HCLK-Mean clustering for different datasets is shown in Table 1.4. It was noted that HCLK Mean clustering's overall purity rises when the choice of attributes includes the relevance of the attributes identified in CHIR. Clustering efficiency decreased when the negative attributes for clustering were included. A tiny rise with negative attributes was noted compared to the technique of choice of $\mathrm{CHI}$ characteristics (Zong 2010). 
Figure 1.8 Comparison of purity of Flocking-based clustering with CHI, CHIR, and CHIR NEG methods for text various datasets

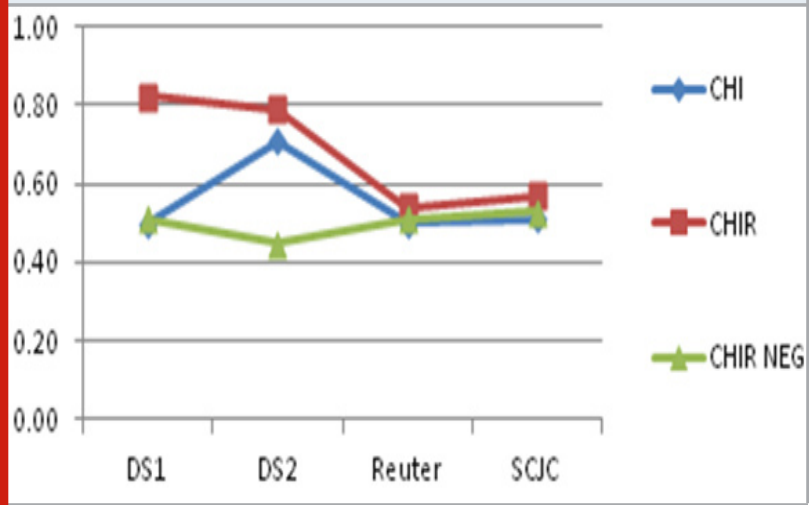

Figure 1.9 Comparison of overall entropy of Flockingbased clustering with $\mathrm{CHI}$, CHIR, and CHIR NEG methods for text various datasets

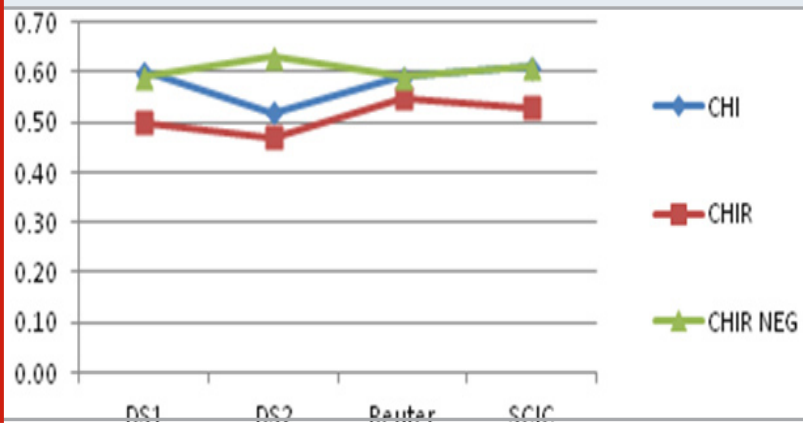

Table 1.5 and Figure 1.5 demonstrate the HCLK-Mean clustering entropy and overall entropy for different datasets. It was noted that HCLK Mean clustering's overall entropy declines when the attribute choice includes the relevance of the attributes identified in CHIR. Clustering efficiency decreased when the negative attributes for clustering were included. There was a tiny rise in efficiency with negative attributes compared to the selection method for CHI attributes.

Table 1.4 Comparison of overall purity of HCLK-Mean clustering with CHI, CHIR, and CHIR NEG methods for text various datasets

\begin{tabular}{|l|c|c|c|}
\hline Dataset & CHI & CHIR & CHIR NEG \\
\hline DS1 & 0.62 & 0.86 & 0.77 \\
\hline DS2 & 0.61 & 0.65 & 0.60 \\
\hline Reuter & 0.53 & 0.72 & 0.64 \\
\hline SCJC & 0.50 & 0.49 & 0.48 \\
\hline
\end{tabular}

1.8.2 Results of the CHI, CHIR, and CHIR NEG with Flocking-based clustering algorithm

Table 1.6 and Table 1.7 demonstrate the precision and recall of flocking-based clustering with CHI, CHIR and
Table 1.5 Comparison of entropy of HCLK-Mean clustering with CHI, CHIR, and CHIR NEG methods for text various datasets

\begin{tabular}{|l|l|l|l|}
\hline Dataset & CHI & CHIR & CHIR NEG \\
\hline DS1 & 0.24 & 0.04 & 0.06 \\
\hline DS2 & 0.37 & 0.20 & 0.23 \\
\hline Reuter & 0.04 & 0.06 & 0.03 \\
\hline SCJC & 0.39 & 0.32 & 0.45 \\
\hline
\end{tabular}

Table 1.6 Comparison of precision of Flocking-based clustering with CHI, CHIR, and CHIR NEG methods for text various datasets

\begin{tabular}{|l|c|c|c|}
\hline Dataset & CHI & CHIR & CHIR NEG \\
\hline DS1 & 0.50 & 0.82 & 0.51 \\
\hline DS2 & 0.71 & 0.79 & 0.45 \\
\hline Reuter & 0.50 & 0.54 & 0.51 \\
\hline SCJC & 0.51 & 0.57 & 0.53 \\
\hline
\end{tabular}

Table 1.7 Comparison of recall of Flocking-based clustering with CHI, CHIR, and CHIR NEG methods for text various datasets

\begin{tabular}{|l|c|c|c|}
\hline Dataset & CHI & CHIR & CHIR NEG \\
\hline DS1 & 0.52 & 0.83 & 0.39 \\
\hline DS2 & 0.81 & 0.81 & 0.36 \\
\hline Reuter & 0.64 & 0.61 & 0.43 \\
\hline SCJC & 0.50 & 0.59 & 0.49 \\
\hline
\end{tabular}

Table 1.8 Comparison of overall purity of Flocking-based clustering with CHI, CHIR, and CHIR NEG methods for text various datasets

\begin{tabular}{|l|l|l|l|}
\hline Dataset & CHI & CHIR & CHIR NEG \\
\hline DS1 & 0.42 & 0.50 & 0.53 \\
\hline DS2 & 0.53 & 0.57 & 0.33 \\
\hline Reuter & 0.39 & 0.47 & 0.42 \\
\hline SCJC & 0.36 & 0.49 & 0.39 \\
\hline
\end{tabular}

CHIR NEG attributes for DS1, DS2, Reuter and SCJC corpus. An increase in clustering precision and recall with the positive attributes chosen by the CHIR method was noted. When negative attributes were in the space of the attribute, there was a drop in both precision and recall for all datasets.

Figure 1.6 illustrates the F-measure of Flocking-based clustering with different attribute selection techniques for the different datasets. When selected attributes were 
observed, it was observed that nouns are related to the dataset to the attributes selected for the different datasets with CHIR. That's why clustering efficiency was always high for all datasets. Attributes selected from datasets were more general terms and were unable to efficiently discriminate against documents.

Figure 1.7 demonstrates overall F-measurement of CHI, CHIR, and CHIR NEG clustering with different text datasets. The clustering algorithm based on Flocking could efficiently cluster the files as each dataset must have a distinctive noun phrase for each dataset category. An increase in Overall F-Measure with all data set with CHIR method's positive attributes.

Figure 1.8 demonstrates the HCLK-Means clustering's relative purity outcomes for different datasets. The clustering of HCLK-Means has been able to efficiently cluster the alt.atheism, comp.graphics, comp.sys.ibm. pc.hardware, comp.sys.mac.hardware, comp.windows.x in DS2 dataset categories. Analysis of the attribute chosen by the CHIR technique showed that between the above categories there were numerous common noun words.

The overall purity of the HCLK-Mean clustering for different datasets is shown in Table 1.8. It was noted that HCLK Mean clustering's overall purity rises when the choice of attributes includes the relevance of the attributes identified in CHIR. Clustering efficiency decreased when the negative attributes for clustering were included. A tiny rise with negative attributes was noted compared to the technique of choice of $\mathrm{CHI}$ attributes.

Table 1.9 Comparison of entropy of Flocking-based clustering with CHI, CHIR, and CHIR NEG methods for text various datasets

\begin{tabular}{|l|l|l|l|}
\hline Dataset & CHI & CHIR & CHIR NEG \\
\hline DS1 & 0.52 & 0.31 & 0.40 \\
\hline DS2 & 0.29 & 0.25 & 0.46 \\
\hline Reuter & 0.18 & 0.16 & 0.41 \\
\hline SCJC & 0.37 & 0.21 & 0.49 \\
\hline
\end{tabular}

Table 1.9 and Figure 1.9 demonstrate the Flocking-based clustering entropy and overall entropy for different datasets. It was noted that the Flocking-based clustering's overall entropy reduces when the attribute selection includes the relevance of the attributes identified in CHIR. Clustering efficiency decreased when the negative attributes for clustering were included. There was a tiny rise in efficiency with negative attributes compared to the selection method for CHI characteristics.

\section{CONCLUSION}

In this article, the clustering performance was analysed using an attribute chosen by the $\mathrm{CHI}$ techniques and BON
Representation was used by CHIR. Then the attribute set included negative attributes recognized by the CHIR technique to analyse their effect on clustering results. It was noted that clustering efficiency increases very well with the incorporation of positive attributes for all datasets using CHIR technique and dropswhen negative features were included. It was observed that when negative attributes were included, there was a slight rise in accuracy than $\mathrm{CHI}$, and also noted that the clustering efficiency differs greatly while working with datasets. It was observed that attributes supplied to tightly associated categories are more essential for clustering documents.

\section{REFERENCES}

Asha Gowda Karegowda, A.S.Manjunath, M.A.Jayaram, (2010)."Comparative study of attribute selection using gain ratio and correlation based feature selection", volume 2, No. 2, Pages: 271 -277, International Journal of Information Technology and Knowledge Management.

Distributed Noun Attribute based on its Compactness for Text Document Clustering, International Journal of Applied Engineering Research, and ISSN: 0973-4562, EISSN 1087-1090, vol.10, No.1 (2015) pp. 403-408 Research India Publication.

Heba Abusamra (2013), “A Comparative Study of Feature Selection and Classification Methods for Gene Expression Data of Glioma”, 4th International Conference on Computational Systems-Biology and Bioinformatics, CSBio2013 , Procedia Computer Science, Volume 23, 2013, Pages 5-14.

Huiqing Liu, Jinyan Li, Lim soon Wong, (2002) "A Comparative Study on Feature Selection and Classification Methods using Gene Expression Profiles and Proteomic Patterns, Genome Informatics 13, Pages: 51-60

IEEE International Conference on Computational Intelligence and Computing Research(2014 IEEE ICCIC), "Distributed Noun Attribute based on its First Appearance for Text Document Clustering" conducted by Park College of Engineering and Technology(18 \& 19 Dec, 2014), Page No. 780-784, ISBN: 978-1-47993974-9. (Scopus)

Jinxin Gaoa David.B, Hitchcock James Stein, (2010) "shrinkage to improve k-means cluster analysis", Journal on Computational Statistics and Data Analysis pages. 2113 - 2127.

Meedeniya D.A and Perera A.S, (2009) "Evaluation of Partition-Based Text Clustering Techniques to Categorize Indic Language Documents”, IEEE International Advanced Computing Conference, (IACC 2009), Digital Object Identifier: 10.1109/ IADCC.2009.4809239, Pages: 1497-1500.

Mitra.P, Murthy.C.A, (2002) "Unsupervised Feature Selection Using Feature Similarity”, IEEE Transactions on Pattern Analysis and Machine Intelligence, 24, pages: 301-312

Ridvan Saracoglu, Kemel Tutuncu, Novruz Allahverdi, 
(2008) "A new Approach on search for similar documents with multiple categories using fuzzy clustering", Expert System with Application, volume 34, pages: 2545-2554.

S.Vijayalakshmi, Dr.D.Manimegalai,(2014) “Text Document Clustering with Flocking Algorithm using Specific Crimes Judgment Corpus", Asian Journal of Information Technology, 13(1):21-28, ISSN: 1682-3915, DOI: 10.3923/ajit.2014.21.28.

S. Vijayalakshmi, Dr.D. Manimegalai, (2013).”Integrating Ontology to Enhance HCL-Based Text Document Clustering”, Research Journal of Applied Sciences, 8(7):358-368, ISSN: 1815-932X,

Salton G., Yang C.S., and Yu C.T. (1975), “A theory of term importance in automatic text analysis, Journal of American society for Information Science, Vol.26, No.1, pp.33-44.

Tao Liu, Sheng ping Liu, Wei-Ying Ma, (2003), "An Evaluation on Feature Selection for Text Clustering", Proceedings of the Twentieth International Conference on Machine Learning (ICML-2003), Washington DC,.

Tzeras and Hartmann S. (1993) "Automatic Indexing based on Bayesian inference networks", Proceedings of SIGIR 93, 16thACM International Conference, Pittsburgh, PA, USA, June27 July 01, 1993, pp22-34. Yanjun Li, CongnanLuo, and Soon M. Chung, Member, IEEE, (2008)“"Text Clustering with Feature Selection by Using Statistical Data”, IEEE transactions on knowledge and data engineering, vol.20, no.5 ,.

Yanjun Li, Congnan Luo, and Soon M. Chung, Member, IEEE,( 2008) "Text Clustering with Feature Selection by Using Statistical Data”, IEEE transactions on knowledge and data engineering, vol.20, no.5.

Yi Hong, Sam Kwong, Yuchou Chang, Qing sheng Ren,( 2007) "Unsupervised Feature Selection Using Clustering Ensembles and Population Based Incremental Learning Algorithm", Preprint submitted to Elsevier.

Yiming Yang and Jan O. Pedersen (1997), "Comparative study of feature selection in Text categorization", Proceedings on Feature Selection in Text Categorization", Proceedings of 14th International conference: Machine learning, Nashville, TN, USA, pp.412-420.

Zong Woo Geem, (2010) "Recent advances in Harmony search algorithm”, Springer science and Business media, ISBN 978-3-642-04316-1. 